LA GRANJA:

REVISTA DE

CIENCIAS DE LA VIDA

pISSN:1390-3799; eISSN:1390-8596

http:/ / doi.org/10.17163/lgr.n27.2018.04
Artículo ciertífico / Scientific paper

ECOLOGÍA FORESTAL

\title{
MEDICIÓN DE CARBONO DEL ESTRATO ARBÓREO DEL BOSQUE NATURAL TINAJILLAS-LIMÓN INDANZA, ECUADOR
}

\section{CARBON MEASUREMENT OF THE NATURAL FOREST ARBOREO STRATUM, TINAJILLAS-LIMON INDANZA}

\author{
Carlos Alberto Jumbo Salazar ${ }^{1,2 *}$, Carla Daniela Arévalo Delgado ${ }^{2}$, \\ Lenin J. Ramirez-Cando ${ }^{3}$
}

\author{
1* Grupo de Investigación en Ecología y Manejo de Recursos Naturales (GIERENA), Universidad Politécnica Salesiana Av. \\ Morán Valverde s/n y Rumichaca, Quito, Ecuador. \\ 2 Fundación Cordillera Tropical \\ ${ }^{3}$ Grupo de Investigación Ambiental para el Desarrollo Sustentable (GIADES), Universidad Politécnica Salesiana Av. Morán \\ Valverde s/n y Rumichaca, Quito, Ecuador
}

*Autor para correspondencia: cajbx@hotmail.com, cjumbo@ups.edu.ec

Artículo enviado el 13 de agosto de 2017. Aceptado, tras revisión el 23 de noviembre de 2017. Publicado el 1 de marzo de 2018.

\begin{abstract}
Resumen
Establecer la eficiencia de los bosques en materia de servicios ecológicos y funciones ecosistémicas, implica descubrir las condiciones que regulan su desempeño, mismas que guardan relación con las características funcionales de las especies vegetales. A fin de aportar con conocimiento sobre estos requerimientos, se condujo la presente investigación, considerando el comportamiento de las especies en función del hábitat, valor de importancia, interacciones intra- e interespecíficas, para con ello, arribar a la determinación de las cantidades de carbono almacenado en el estrato arbóreo del bosque natural Tinajillas, ubicado al sur de la Cordillera Oriental de los Andes, cantón Limón Indanza, provincia de Morona Santiago. Para el efecto, se aplicó el método recomendado por el Manual de Campo de la Evaluación Nacional Forestal del Ecuador, y los criterios del Panel Intergubernamental para el Cambio climático. Como resultado de la investigación, se estableció que en las 118 ha de bosque, el volumen de madera es de $13521 \mathrm{~m}^{3}$, es decir $115 \mathrm{~m}^{3} / \mathrm{ha}$, representado en su mayoría por especies de la familia Melastomataceae, entre las que consta Miconia sp. La cantidad de carbono almacenado, es de $4835 \mathrm{t}$, resultando $41 \mathrm{t} / \mathrm{ha}$. La familia Melastomataceae contiene mayor cantidad de carbono (13t/ha), mientras que la especie con mayor cantidad de carbono almacenado es Miconia sp con un valor de $8 \mathrm{t} / \mathrm{ha}$. Al resultar los índices de valor de importancia en favor de estas especies y juzgando por las Clases Naturales de Edad, se desprende que el bosque se encuentra en un período de sucesión vegetal, situación que contribuye a generar una importante dinámica en materia de captura de carbono.

Palabras claves: bosques, cambio climático, carbono, especies forestales, gases de efecto invernadero.
\end{abstract}

LA GRANJa:Revista de Ciencias de la Vida 27(1) 2018:51-63.

(C)2018, Universidad Politécnica Salesiana, Ecuador. 


\begin{abstract}
To stablish the efficiency of the forest in the subject of ecological services and ecosystems functions, involve to discover the conditions to set its performance, which ones that keep the relationship with the functional characteristics of the vegetable species, in order to contribute with knowledge about this requirements, it takes the present investigation, considering the behavior of the species in the performance of the environment, or habitat, important value, interactions, intra- and interspecific, in order to reach and determinate the quantity of the stored carbon in the arboreal stratum of the natural forest Tinajillas, located in the south east Andes range in the canton Limon Indanza, Morona Santiago province, in order to reach it, we apply the suggested method for the support manual of the National evaluation of Ecuador, and the professional forest judgment of the Intergovernmental Panel on Climate Change, with this results of the investigation, we stablish that in the 118 ha forest, the volume of the timber is $13570 \mathrm{~m}^{3}$, it means 115 $\mathrm{m}^{3} /$ ha represented in the majority of the Family species Melastomataceae, in this one is the Miconia sp. The quantity of the stored carbon is 4835 tones, and the result is 41 tones per hectare. The Family, Melastomataceae has more quantity of carbon (13 tones per hectare), then the specie with more quantity of stored carbon is Miconia sp, with a value of 8 $t /$ ha the result of the indices of the important value in favor of this species and judging for the edge of the natural classes, it understands that the forest is in a period of the vegetable sucetion, situation that contribute to generate an important dynamic in the way to capture Carbon.
\end{abstract}

Keywords: Forest, climate change, Carbon, Forestry species, gases of the greenhouse efect.

Forma sugerida de citar: Jumbo Salazar, C., Arévalo Delgado, C. D. y Ramirez-Cando, L. J. 2018. Medición de carbono del estrato arbóreo del bosque natural Tinajillas-Limón Indanza, Ecuador. La Granja: Revista de Ciencias de la Vida. Vol. 27(1):51-63. http://doi.org/10.17163/lgr. n27.2018.04. 


\section{Introducción}

El cambio climático, es un fenómeno que altera el comportamiento de los distintos ecosistemas a nivel global. Se atribuye este cambio a actividades humanas y a causas naturales, destacando un predominio de las primeras; a partir del cual, son varios los efectos que se derivan, como el incremento en los eventos climáticos, éstos son las sequías y precipitaciones intensas (Riebeek, 2005). Por su parte, los gases de efecto invernadero (GEI) también aumentan, principalmente el dióxido de carbono $\left(\mathrm{CO}_{2}\right)$ producido por las actividades humanas, consumo de combustibles fósiles, deforestación y cambio de uso del suelo. A su vez, existen algunas particularidades que generan el cambio climático como: el incremento general y gradual de la temperatura, cambios en los comportamientos de las precipitaciones e incremento de eventos extremos (IPCC, 1995).

Una forma de mitigar estos efectos consiste en la capacidad de absorción de $\mathrm{CO}_{2}$ de la atmósfera y fijar el carbono en la biomasa de los bosques, lo cual sucede por medio del proceso de fotosíntesis (FAO, 2006; Yáñez Sandoval, 2004). Por tanto, los bosques juegan un rol importante, tomando en cuenta que la vegetación y el suelo intercambian aproximadamente el $80 \%$ de carbono con la atmósfera. Gracias a este proceso almacenan cantidades de carbono en la biomasa de sus hojas, ramas, tallos y raíces, mientras liberan oxígeno hacia la atmósfera, actuando como sumideros de carbono (Pardos, 2010).

El carbono almacenado, se encuentra en la biomasa de los árboles y corresponde a la materia orgánica producida en un bosque, destacándose cuatro tipos de biomasa: biomasa viva, biomasa subterránea, materia orgánica muerta y biomasa en el suelo (FAO, 2002), variables que son consideradas en la presente investigación, a fin de determinar los volúmenes de carbono almacenado en los ecosistemas de bosques.

La importancia de realizar este tipo de mediciones en los bosques naturales para determinar el carbono almacenado radica en que éstos cumplen un papel crucial en el ambiente por los servicios que ellos ofrecen. Dentro de los más significativos están: la protección de cuencas hidrográficas, servicios hidrológicos, captura de carbono, belleza paisajística, biodiversidad (Robertson and Wunder, 2005).

\section{Materiales y métodos}

\subsection{Ubicación del área de estudio}

El bosque está ubicado al sur de la Cordillera Oriental de los Andes, en el cantón Limón Indanza, provincia de Morona Santiago, contiene 118 ha y comprende las formaciones vegetales Bosque siempre verde montano y Bosque siempre verde montano bajo.

\subsection{Estratificación del área de estudio}

El área se delimitó a partir de mapas topográficos. El perímetro se definió por georreferenciación, utilizando herramientas del Sistema de Información Geográfica y cartografía, apoyada con equipamientos GPS, interpretación de imágenes de satélites y fotos aéreas. Para la estratificación se aplicó la "Guía para la determinación de carbono en pequeñas propiedades rurales" (Rügnitz Tito, Chacón León and Porro, 2009)) que considera los factores fundamentales como: áreas con similares prácticas de manejo e historial del uso del suelo, características del suelo, microclima, relieve, especies de árboles existentes, estado de madurez del bosque, entre otros, mismos que inciden en la cantidad de carbono, estratificación preliminar y elaboración del mapa de estratificación del área.

\subsection{Determinación del diseño del sitio de muestreo}

Para la definición del tipo y diseño de muestreo se procedió de la manera siguiente:

\subsubsection{Tipo de parcela}

Se recurrió a la técnica de muestreo, instalando Parcelas Permanentes de Muestreo (PPM), por ser más eficientes y para realizar mediciones futuras (Corral-Rivas et al., 2013), a fin de ir determinando la dinámica del bosque en su estado natural, respecto de la fijación de carbono.

\subsubsection{Diseño de muestreo}

Se instaló un conglomerado, conformado por 4 parcelas de $60 \times 60 \mathrm{~m}$, localizadas en forma de "L" (Figura 1), dando una superficie de $14400 \mathrm{~m}^{2}$, distanciadas entre ellas a $250 \mathrm{~m}$. El conglomerado, fue implementado para una superficie de $100 \mathrm{ha}$, como in- 
dica el Manual de Campo para la Evaluación Nacio- nal Forestal 2012 (MAE, 2012).
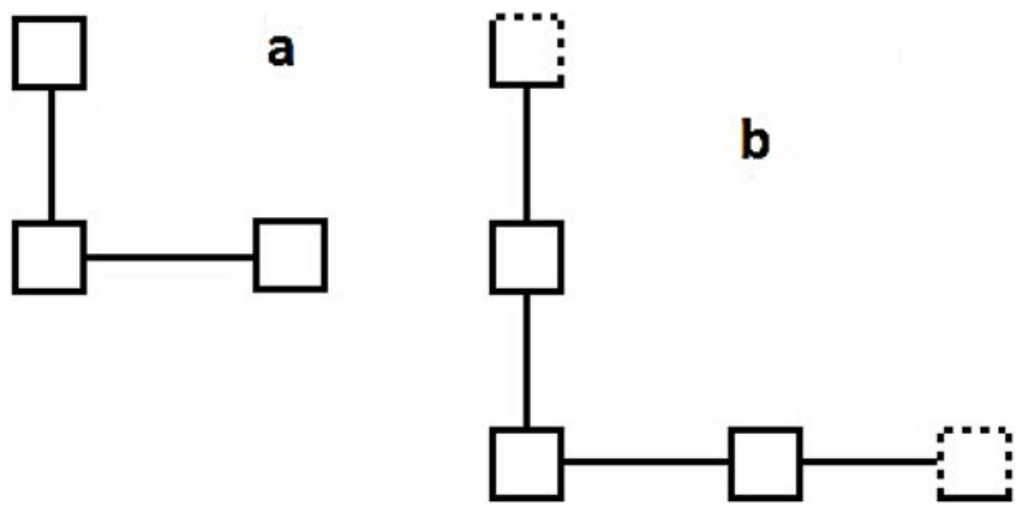

Figura 1. Diseño de conglomerado en forma de "L"

\subsubsection{Medición de las parcelas}

Las parcelas fueron medidas con respecto a un plano horizontal. Debido a que el terreno cuenta con una topografía irregular, se aplicaron los Factores de Corrección de Pendientes, establecidas por la Organización de las Naciones Unidas para la Agricultura y la Alimentación-FAO (FAO, 2004). Las parcelas fueron instaladas aplicando el programa Arcgis y GPS, con lo cual se identificó la ubicación y se determinó el punto central de cada parcela a fin de tener la referencia para establecer la forma y los límites de cada unidad de medida.

\subsubsection{Instalación de las parcelas}

Las parcelas, se proyectaron utilizando el método de faja o carril (FAO, 2015a) como se muestra en la Figura 1. Estas parcelas fueron subdivididas en 3 carriles de 20 × $60 \mathrm{~m}$ cada uno.

\subsubsection{Efecto de borde de las parcelas}

Para evitar el efecto de borde, se midieron los árboles que se encontraron dentro de la parcela, a partir de los $10 \mathrm{~m}$ de su eje central y no se consideraron los árboles ubicados fuera de esta distancia, con ello, se aseguró que los árboles no se encuentren cerca del límite externo del bosque.

\subsection{Mensuración forestal}

El DAP fue medido con corteza a 1,30 m, a partir de $10 \mathrm{~cm}$. La medición consistió en iniciar desde el carril 1 de la primera subparcela de $20 \times 60 \mathrm{~m}$.

Para determinar la biomasa, carbono y $\mathrm{CO}_{2}$, se realizaron las siguientes estimaciones:

\subsubsection{Estimación del área basal}

El área basal se calculó aplicando la siguiente fórmula:

$$
A B=\frac{\pi}{4} \times D A P^{2}
$$

Donde:

$\mathrm{AB}=$ área basal, $\mathrm{m}^{2}$.

$\frac{\pi}{4}=$ constante 0,7854 .

$\mathrm{DAP}^{2}=$ diámetro a la altura el pecho, $\mathrm{m}$

\subsubsection{Estimación del volumen}

El volumen se determinó mediante la siguiente fórmula:

$$
\text { Volumen }=A B \times H \times f f
$$

Donde:

$\mathrm{AB}=$ área basal, $\mathrm{m}^{2}$.

$\mathrm{H}=$ altura total del árbol, $\mathrm{m}$.

$\mathrm{ff}=$ factor de forma (Latifoliadas: 0,5 ).

\subsubsection{Estimación de la biomasa forestal}

La biomasa es un elemento principal para determinar la cantidad de carbono almacenado en el bosque 
(FAO, 2015b). La biomasa forestal, da a conocer el ciclo del carbono a nivel global, por lo que constituye un elemento importante para estudios de cambio climático. Esta estimación se obtuvo de la multiplicación del volumen del bosque, la densidad de la madera, el factor de expansión de biomasa aérea y el factor de expansión de biomasa subterránea.

Acorde a las Directrices del Panel Intergubernamental para el Cambio Climático, el factor de expansión aplicado fue de 1,20 para la biomasa aérea y subterránea (IPCC, 2006). El valor empleado para la densidad de madera fue 0,5 (Brown and FAO. 1997; IPCC, 2006). El valor de la biomasa, se obtuvo mediante la siguiente formula:

$$
B f=\text { Volumen } \times G E \times F E B a \times F E B s
$$

Donde:

$\mathrm{Bf}=$ biomasa forestal, $\mathrm{t}$.

$\mathrm{GE}=$ densidad de la madera, $\mathrm{t} / \mathrm{m}^{3}[0,5]$.

$\mathrm{FEBa}=$ factor de expansión de biomasa aérea (ramas,hojas) [1,20].

FEBs=factor de expansión de biomasa subterránea (raices) [1,20].

\subsubsection{Estimación del carbono almacenado en el bos- que}

El carbono almacenado, se calculó a partir de los datos de biomasa forestal del área y se aplicó la fracción de carbono de 0,5 , asumiendo que el $50 \%$ del peso de los individuos es carbono (IPCC, 1995). La fórmula para la obtención del valor de carbono almacenado es:

$$
C=B \times F c
$$

Donde:

$\mathrm{C}=$ carbono en toneladas de carbono.

$\mathrm{B}=$ biomasa

$\mathrm{Fc}=$ fracción de carbono $[0,5]$.

\subsubsection{Estimación del $\mathrm{CO}_{2}$ almacenado en el bosque}

Una tonelada de carbono equivale al secuestro de 3,67 t de $\mathrm{CO}_{2}$. Para establecer la cantidad de $\mathrm{CO}_{2}$ almacenado a partir de la cantidad de carbono se aplicó la fórmula:

$$
\mathrm{CO}_{2}=\mathrm{C} \times 3,67
$$

Donde:

$\mathrm{CO}_{2}=$ cantidad de dióxido de carbono capturado en toneladas de $\mathrm{CO}_{2}$.

3,67 = fracción que se utiliza para convertir carbono en toneladas de $\mathrm{CO}_{2}$.

\subsection{Identificación de especies}

\subsubsection{Recolección de muestras}

Las muestras fueron recolectadas y codificadas en el campo, con nombre común y especificaciones. Se recolectaron muestras en estado fértil con flores o frutos para facilitar la identificación. Como parte de la recolección, se desarrolló un registro fotográfico.

\subsubsection{Tratamiento y determinación de muestras}

Las muestras de las especies recolectadas fueron puestas en una prensa y ésta a su vez fue colocada en una cámara de secado. La identificación de las muestras se realizó con el apoyo del Herbario de la Universidad del Azuay (UDA).

\section{6 Índice de valor de importancia por es- pecie}

El Índice de Valor de Importancia por especie, es un índice sintético estructural, desarrollado principalmente para jerarquizar la dominancia de cada especie en rodales. El IVI puede fluctuar de 0 a 3.00 (o $300 \%$ ). Al dividir el IVI por 3, se obtiene una cifra que fluctúa de 0 a 1.00 (o $100 \%$ ). Este valor se conoce como el porcentaje de importancia. El valor de importancia, o el porcentaje de importancia, provee un estimado global de la importancia de una especie de planta en una comunidad determinada (Zarco-Espinoza et al., 2010), la fórmula es:

$$
\begin{array}{r}
I V I=\text { Dominancia relativa }+ \text { Densidad relativa }+ \\
+ \text { Frecuencia relativa }
\end{array}
$$

\section{Resultados y Discusión}

\subsection{Composición del estrato arbóreo}

El bosque natural Tinajillas, se encuentra en un proceso activo de sucesión, como reacción a las constantes perturbaciones de carácter tanto natural como antropogénico. En el primer caso, las razones 
obedecen a que el bosque al encontrase en estribaciones pronunciadas, está sujeto a frecuentes deslizamientos. El carácter antropogénico, se debe a que hay fuertes presiones por procesos de colonización, implica que el estrato arbóreo del bosque cuenta con individuos que están superando la fase de latizales, hacia arbolitos. Las especies existentes por familia, se indica en la Tabla 1.

Tabla 1. Especies existentes por familia

\begin{tabular}{|c|c|c|}
\hline \multicolumn{3}{|c|}{ ESPECIES EXISTENTES POR FAMILIA } \\
\hline FAMILIA & NOMBRE COMÚN & NOMBRE CIENTÍFICO \\
\hline Araliaceae & Pumamaqui & Schefflera morototoni (Aubl.) \\
\hline Asteraceae & Negrillo & Critoniopsis sp. \\
\hline Brunelliaceae & Bella maría & Brunellia sp. \\
\hline Chloranthaceae & Pururu & Hedyosmum cuatrecazanum Occhioni \\
\hline \multirow{2}{*}{ Clusiaceae } & Achotillo & Vismia acuminata (Lam.) Pers \\
\hline & Duco & Clusia latipes Planch. \& Triana \\
\hline Cunoniaceae & Sarar & Weinmannia pinnata Linnaeus \\
\hline Ericaceae & Joyapa & Cavendishia bracteata Ruiz \& Pav. \\
\hline Escalionacea & Capulí & Escallonia paniculata (Ruiz \& Pav.) Roem. \& Schult \\
\hline \multirow{2}{*}{ Euphorbiaceae } & Motilón & Hyeronima alchornoides Allemão \\
\hline & Palo del diablo & Alchornea latifolia Swartz \\
\hline Humiriaceae & Chanul & Humiriastrum sp. \\
\hline \multirow{2}{*}{ Lauraceae } & Canelo & Ocotea javitensis (Kunth) Pittier \\
\hline & Jigua & Nectandra reticulata (Ruiz \& Pav.) Mez \\
\hline \multirow{2}{*}{ Melastomataceae } & Flor rosada & Tibouchina lepidota Cogniaux \\
\hline & Palo de agua & Miconia sp. \\
\hline Melastomataceae & Poma rosa & Blakea grandulosa Gleason. \\
\hline Rutaceae & Limoncillo & Zanthoxylum ekmanii (Urb.) Alain \\
\hline Meliaceae & Sacha coco & Guarea kunthiana A. Juss. \\
\hline Moraceae & Guarumbo & Cecropia sciadophylla Martius \\
\hline Myricaceae & Laurel & Myrica pubescens Humb. \& Bonpl. ex Willd. \\
\hline Myrsinaceae & Jiripe & Myrsine andina (Mez) Pipoly \\
\hline Myrtaceae & Chimulo & Eugenia sp. \\
\hline Podocarpaceae & Guabisay & Podocarpus oleifolius D. Don ex Lamb. \\
\hline Rubiaceae & Cebolla & Elaeagia $s p$ \\
\hline Staphyleaceae & Facte & Turpinia occidentalis (Sw.) G. Don \\
\hline Bombacaceae & Higeron & Matisia sp. \\
\hline
\end{tabular}


La composición interespecífica del ensayo que cubre una superficie de $14400 \mathrm{~m}^{2}$, está determinada por 925 individuos, agrupados en 27 especies, 27 géneros y 22 familias y presenta un amplio rango de individuos por especie, tal es el caso de Miconia sp que arroja 323 individuos (35\% del total), mientras que 3 especies como Blakea grandulosa, Escallonia paniculata, Matisia sp, cuentan con 1 individuo. Las especies Brunellia sp y Cavendishia bracteata, cuentan con 2 individuos por ha, lo que demuestra que las referidas especies constituyen un indicador de rareza.

\section{2 Índice de valor de importancia por es- pecie}

Las 5 especies con mayor Índice de Valor de Importancia se indican en la Tabla 2.

Las especies más abundantes son: Miconia sp con 323 individuos, Weinmannia pinnata con 86 individuos, Clusia latipes con 72 individuos, Ocotea javitensis con 56 individuos y Zanthoxylum ekmanii con 52 individuos.

Considerando el índice de valor de importancia (IVI), la especie más dominante es Miconia sp con un valor de IVI igual al 21\%, Weinmannia pinnata y Clusia latipes con un IVI igual al 7\%, Ocotea javitensis y Zanthoxylum ekmanii con un IVI del 6\%. Las demás especies no superan el $5 \%$ de IVI.

Tabla 2. Especies existentes por familia

\begin{tabular}{ccccccccc}
\hline \multicolumn{1}{c}{ ÍNDICE DE VALOR DE IMPORTANCIA (IVI) POR ESPECIE } \\
\hline ESPECIE & Aa & Ar \% & Fa & Fr \% & Da & Dr \% & IVI 300 \% & IVI 100\% \\
\hline Miconia sp. & 323 & 34,92 & 3 & 4,35 & 6,15 & 23,22 & 62 & 21 \\
\hline Weinmannia pinnata Linnaeus. & 86 & 9,3 & 3 & 4,35 & 1,95 & 7,35 & 21 & 7 \\
\hline Clusia latipes Planchon. \& Triana & 72 & 7,78 & 3 & 4,35 & 2,27 & 8,57 & 21 & 7 \\
\hline Ocotea javitensis (Kunth) Pittier & 56 & 6,05 & 3 & 4,35 & 2,27 & 8,57 & 19 & 6 \\
\hline Zanthoxylum ekmanii (Urb.) Alain & 52 & 5,62 & 3 & 4,35 & 1,82 & 6,86 & 17 & 6 \\
\hline $\begin{array}{l}\text { Abundancia absoluta (Aa), Abundancia relativa (Ar\%), Frecuencia absoluta (Fa), Frecuencia relativa } \\
\text { (Fr\%), Dominancia absoluta (Da), Dominancia relativa (Dr\%) }\end{array}$ \\
\hline
\end{tabular}

\subsection{Análisis de datos}

\subsection{1 Área basal, $\mathrm{m}^{2}$}

El área basal que contiene el bosque en su totalidad es de $2131 \mathrm{~m}^{2}$, lo que representa un valor de $18 \mathrm{~m}^{2} /$ ha. A nivel de familia, las especies con mayor área basal pertenecen a Melastomataceae con un valor de $8,40 \mathrm{~m}^{2}$, es decir el $32 \%$ del total de las unidades de medición. De la misma forma la especie con mayor área basal es Miconia sp con un valor de $6,15 \mathrm{~m}^{2}$, que corresponde al $24 \%$ del total de especies forestales.

\subsubsection{Volumen, $\mathbf{m}^{3}$}

El volumen total de madera del área de estudio tiene un valor de $13521 \mathrm{~m}^{3}$, lo que equivale a 115 $\mathrm{m}^{3} /$ ha. El mayor volumen, se muestra en la familia Melastomataceae con un valor de $49,70 \mathrm{~m}^{3} / \mathrm{ha}$ lo que representa un $30 \%$, mientras que la especie con mayor volumen es Miconia sp 33,60 $\mathrm{m}^{3}$ / ha, es decir, el $20 \%$ del total.

Para la clasificación diamétrica, los diámetros fueron distribuidos en intervalos de $5 \mathrm{~cm}$ generando 9 categorías, cuyos resultados se muestran en la Tabla 3 y Figura 1: 
Tabla 3. Número de individuos y su volumen por clase diamétrica

\begin{tabular}{cccc}
\hline \multicolumn{4}{c}{ CLASES DIAMÉTRICA } \\
\hline CLASE & RANGO & INDIVIDUOS & VOLUMEN, $\mathbf{~ m}^{3}$ \\
\hline I & $\geq 10-15 \mathrm{~cm}$ & 514 & 26,94 \\
II & $\geq 15,1-20 \mathrm{~cm}$ & 191 & 25,02 \\
III & $\geq 20,1-25 \mathrm{~cm}$ & 98 & 25,34 \\
IV & $\geq 25,1-30 \mathrm{~cm}$ & 52 & 20,81 \\
V & $\geq 30,1-35 \mathrm{~cm}$ & 36 & 20,88 \\
VI & $\geq 35,1-40 \mathrm{~cm}$ & 15 & 12,17 \\
VII & $\geq 40,1-45 \mathrm{~cm}$ & 8 & 9,3 \\
VIII & $\geq 45,1-50 \mathrm{~cm}$ & 5 & 8,22 \\
IX & $\geq 50,1 \mathrm{~cm}$ & 6 & 16,42 \\
TOTAL & & 925 & 165 \\
\hline
\end{tabular}

La Tabla 3, expresa que la mayor cantidad de individuos, es decir 514 (56\%), se encuentran en la clase I, dando un volumen de $26,94 \mathrm{~m}^{3}$. La clase II engloba 191 individuos con un volumen de $25,02 \mathrm{~m}^{3}$; por su parte, la clase III con 98 individuos arroja un volumen de $25,34 \mathrm{~m}^{3}$. Las tres primeras clases diamétricas que cubren el rango de 10 a $25 \mathrm{~cm}$ de DAP, cuentan con 803 individuos (87\%) y arrojan un volumen de 77,30 $\mathrm{m}^{3}$ (47\%). El menor volumen se concentra en la clase IX con 16,42 $\mathrm{m}^{3}$. Estos resultados,

\section{Distribucion DAP}

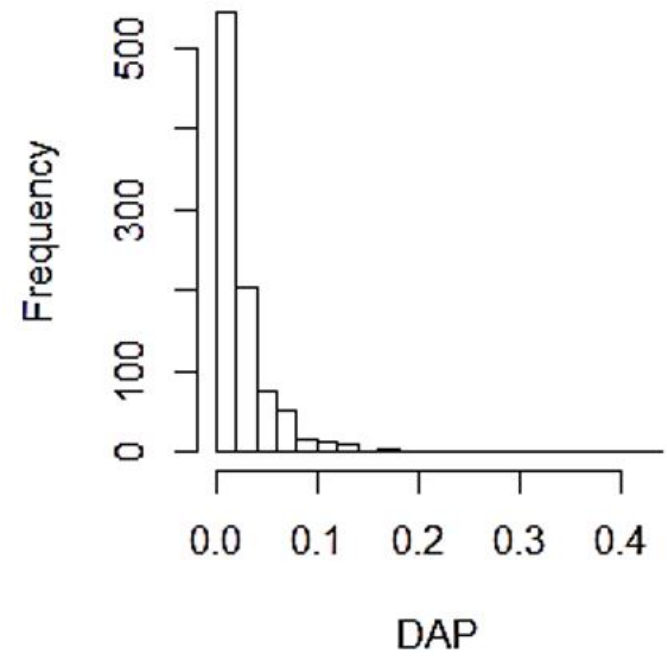

confirman que el bosque se encuentra en un proceso activo de sucesión vegetal, en el cual Miconia sp tiene un rol preponderante.

\subsubsection{Biomasa forestal}

La biomasa forestal total es de $9751 \mathrm{t}$, resultando un valor de $83 \mathrm{t} / \mathrm{ha}$. La familia con mayor biomasa forestal es Melastomataceae con un valor de 36 t (30\%). La especie con una cantidad importante de biomasa es Miconia sp, con $24 \mathrm{t}$, indicando un $20 \%$ del total.

Figura 2. Relación de la distribución entre DAP y altura 


\subsubsection{Carbono almacenado}

El total del carbono almacenado abarca $4835 \mathrm{t} / \mathrm{ha}$ $\mathrm{y}$ el carbono almacenado es de $41 \mathrm{t} / \mathrm{ha}$. La familia más representativa es Melastomataceae con una cantidad de $18 \mathrm{t}$ lo que corresponde el $31 \%$. La especie con mayor cantidad de carbono almacenado es $\mathrm{Mi}$ conia sp, con un valor de $12 \mathrm{t}$, resultando el $20 \%$ de las unidades de medición del carbono almacenado.

\subsection{5 $\mathrm{CO}_{2}$ almacenado}

En el área de estudio el $\mathrm{CO}_{2}$ almacenado total es de 17864 t, indicando una cantidad de 151 t/ha. La fa- milia con más cantidad de $\mathrm{CO}_{2}$ almacenado es $\mathrm{Me}$ lastomataceae con $66 \mathrm{t}$ representando el 30\% del área de estudio. La especie con mayor contenido de $\mathrm{CO}_{2}$ almacenado es Miconia sp, con un valor de $44 \mathrm{t}$, lo que significa un $20 \%$ del total existente en las unidades de medición de $\mathrm{CO}_{2}$, que responde al gran número de individuos presentes en la zona de estudio. Por otro lado, como se observa en la Figura 3, el comportamiento dentro del estudio en términos de carbono y dióxido de carbono almacenado es heterogéneo.

\section{Carbono por especie}

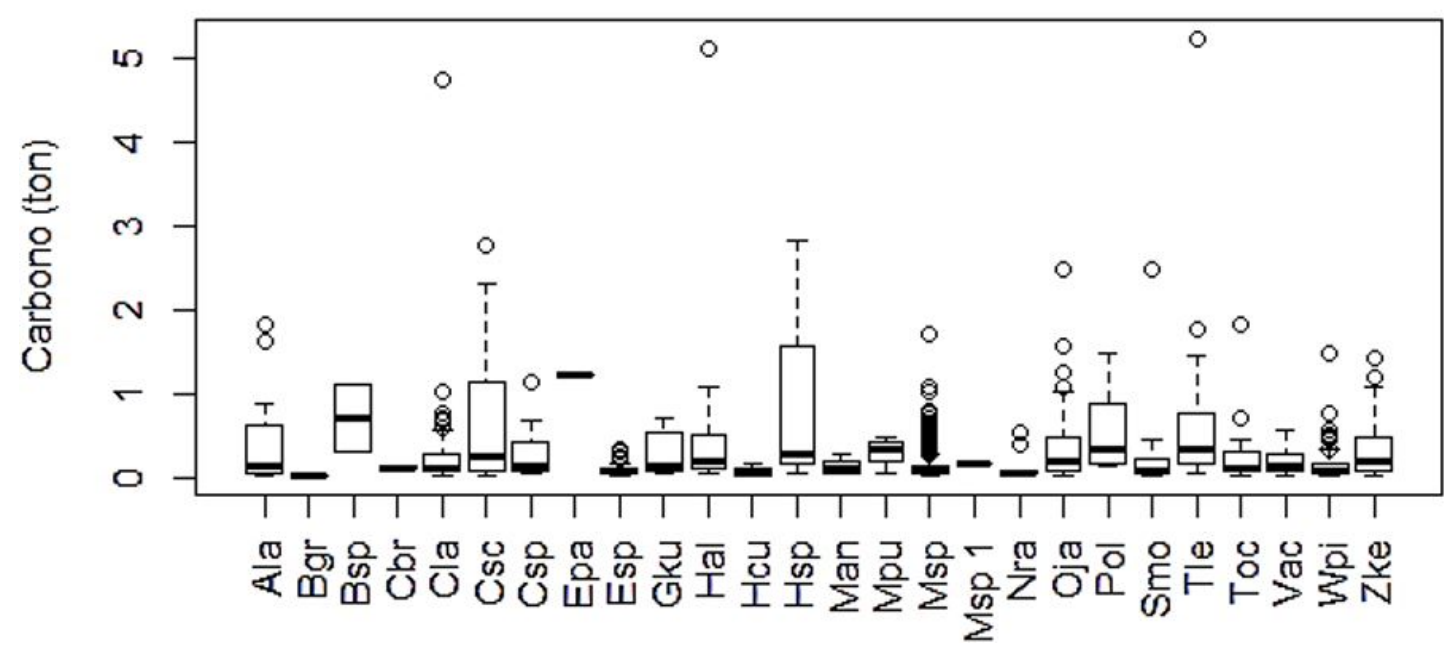

Figura 3. Boxplot del carbono almacena por especie, siguiendo la abreviatura expresada en la Tabla 1

\subsection{Análisis estadístico de la estimación}

En el análisis de la relación entre las variables DAP y la altura total del fuste de los árboles y la biomasa o carbono almacenado, se modeló siguiendo las fórmulas expresadas en la metodología y se corrobora con el comportamiento de la biomasa como variable dependiente del DAP.

$$
\text { Biomasa } \simeq \alpha e^{\beta \times \ln (D A P)}
$$

Donde alfa y beta son coeficientes a estimar a partir de los datos, los cuales explican la relación entre la biomasa (carbono o $\mathrm{CO}_{2}$ ) y el DAP, según Mora et al. (2017), las estimaciones de este tipo tienen el comportamiento antes mencionado. 


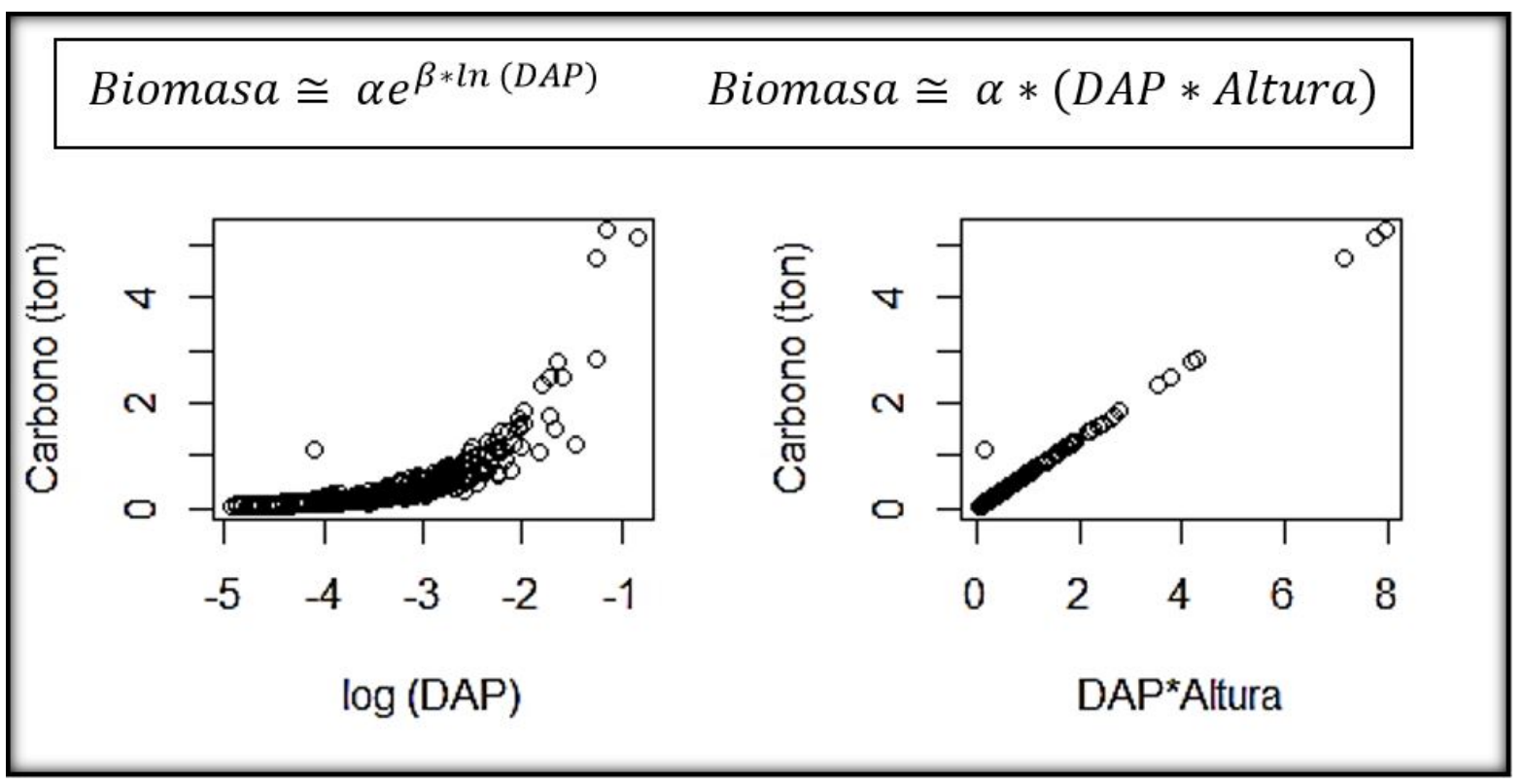

Figura 4. Gráficas de la estimación de carbono según las fórmulas expresadas

No existe una relación directa entre DAP y la altura del fuste, observable en la Figura 2. Particularmente DAP sigue una distribución lognormal por lo cual no se puede estimar una relación directa, sin embargo, es explicado con un coeficiente casi igual a 1, la relación entre la biomasa y la interacción entre DAP y altura como se muestra en la Figura 4. El análisis del modelo se muestra en la Tabla 5.

Tabla 4. Estimación de los modelos presentados en la Figura 4.

\begin{tabular}{ccccc}
\hline & \multicolumn{2}{c}{$\begin{array}{c}\text { Biomasa } \simeq \boldsymbol{\alpha} \times(\text { DAP } \times \text { Altura }) \\
\text { Intercepto }\end{array}$} & $\begin{array}{c}\text { Biomasa } \simeq \alpha e^{\beta \times \ln (\boldsymbol{D A P})} \\
\text { DAP } \times \text { Altura }\end{array}$ & $\begin{array}{ccc}\text { Intercepto } \\
\ln (\mathrm{DAP})\end{array}$ \\
\hline Estimación & 0,001249 & 0,660099 & 2,74546 & 1,22168 \\
Error std & 0,0012 & 0,0017 & 0,0404 & 0,0101 \\
Probabilidad & 0,31 & $2,00 \mathrm{E}-16$ & $2,00 \mathrm{E}-16$ & $2,00 \mathrm{E}-16$ \\
p-value (modelo) & & $2,20 \mathrm{E}-16$ & $2,20 \mathrm{E}-16$ \\
R2 & & 0,994 & 0,9406 \\
\hline
\end{tabular}

\subsection{1 Índices - análisis de biodiversidad}

Los índices utilizados en el análisis de la medición de la biodiversidad son: Shannon - Weinner, Margalef y Simpson, De acuerdo a los valores obtenidos del análisis de los tres índices, existe una relación entre ellos, en razón que el resultado de mayor biodiversidad y riqueza coincide entre dichos índices. Las 3 especies con los índices más abundantes de diversidad son Miconia sp, Weinmannia pinnata, Clusia latipes, como se muestra a continuación: 
Tabla 5. Estimación de los modelos presentados en la Figura 4.

\begin{tabular}{|c|c|c|c|c|}
\hline \multicolumn{5}{|c|}{ ANÁLISIS ESTADÍSTICO PARA BIODIVERSIDAD DE ESPECIES } \\
\hline \multirow[b]{2}{*}{ Especie } & \multirow[b]{2}{*}{ Individuos } & \multicolumn{3}{|c|}{ ÍNDICES } \\
\hline & & $\begin{array}{l}\text { Shannon - } \\
\text { Wienner }\end{array}$ & Margalef & Simpson \\
\hline Miconia sp. & 323 & 0,53 & 109 & 8 \\
\hline Weinmannia pinnata Linnaeus. & 86 & 0,32 & 29 & 116 \\
\hline Clusia latipes Planch. E Triana & 72 & 0,29 & 24 & 165 \\
\hline Ocotea javitensis (Kunth) Pittier & 56 & 0,24 & 19 & 273 \\
\hline Zanthoxylum ekmanii (Urb.) Alain & 52 & 0,23 & 17 & 316 \\
\hline Elaeagia $s p$ & 43 & 0,21 & 14 & 463 \\
\hline Tibouchina lepidota Cogniaux & 34 & 0,18 & 11 & 740 \\
\hline Hyeronima alchornoides Allemão & 33 & 0,17 & 11 & 786 \\
\hline Turpinia occidentalis (Sw.) G. Don & 31 & 0,16 & 10 & 890 \\
\hline Schefflera morototoni (Aubl.) Maguire, Steyerm. \& Frodin & 30 & 0,16 & 10 & 951 \\
\hline Alchornea latifolia Swartz & 26 & 0,14 & 8 & 1266 \\
\hline Hedyosmum cuatrecazanum Occhioni & 26 & 0,14 & 8 & 1266 \\
\hline Vismia acuminata (Lam.) Pers. & 24 & 0,14 & 8 & 1485 \\
\hline Critoniopsis $s p$ & 18 & 0,11 & 6 & 2641 \\
\hline Myrsine andina (Mez) Pipoly & 13 & 0,09 & 4 & 5063 \\
\hline Nectandra reticulata (Ruiz \& Pav.) Mez & 11 & 0,08 & 3 & 7071 \\
\hline Cecropia sciadophylla Martius & 9 & 0,07 & 3 & 10563 \\
\hline Myrica pubescens Humb. E Bonpl. ex Willd. & 9 & 0,07 & 3 & 10563 \\
\hline Podocarpus oleifolius D. Don ex Lamb. & 7 & 0,05 & 2 & 17462 \\
\hline Guarea kunthiana A. Juss. & 7 & 0,05 & 2 & 17462 \\
\hline Humiriastrum sp. & 4 & 0,03 & 1 & 53477 \\
\hline Eugenia sp. & 4 & 0,03 & 1 & 53477 \\
\hline Brunellia sp & 2 & 0,02 & 0 & 213906 \\
\hline Cavendishia bracteata (Ruiz \& Pav. ex J. St.-Hil.) Hoerold & 2 & 0,02 & 0 & 213906 \\
\hline Blakea grandulosa Gleason & 1 & 0,01 & 0 & 855625 \\
\hline Escallonia paniculata (Ruiz \& Pav.) Roem. \& Schult. & 1 & 0,01 & 0 & 855625 \\
\hline Matisia sp. & 1 & 0,01 & 0 & 855625 \\
\hline Número de individuos & 925 & & & \\
\hline
\end{tabular}

\section{Conclusiones}

La flora del bosque natural Tinajillas - Limón Indanza, está compuesta por una alta diversidad, re- presentada por 22 familias, 27 géneros y 27 especies. Las familias con más de una especie son: Clusiaceae, Euphorbiaceae, Lauraceae, Melastomataceae; las familias con mayor influencia son Melastomataceae, $\mathrm{Clu}$ - 
siaceae, Cunoniaceae, Lauraceae y Euphorbiaceae. Con respecto al género, el más abundante es Miconia, le siguen Weinmannia y Clusia. En cuanto a las especies predominantes, éstas son: Miconia sp., Weinmannia pinnata, Clusia latipes.

El volumen de madera existente en el estrato arbóreo del bosque natural Tinajillas - Limón Indanza es de $115 \mathrm{~m}^{3}$ / ha, representado en su mayoría por la familia Melastomataceae y la especie Miconia sp con $35 \mathrm{~m}^{3}$ / ha y $23 \mathrm{~m}^{3} /$ ha respectivamente.

Con respecto al DAP, se presentaron 9 clases diamétricas, dando como resultado que la clase I, que comprende entre $\geq 10-15 \mathrm{~cm}$, es la que mayor número de individuos y volumen de madera arroja, clase que conforma la mayor parte de los 514 individuos, resultando de estos un volumen de 18,68 $\mathrm{m}^{3} /$ ha

La cantidad de carbono almacenado en el área de estudio del bosque natural Tinajillas-Limón Indanza, contiene $4835 \mathrm{t}$, resultando $41 \mathrm{t} / \mathrm{ha}$. La familia con mayor cantidad es Melastomataceae con 13 t/ha. Así mismo, la especie con mayor cantidad de carbono almacenado es Miconia sp. con un valor de $8 \mathrm{t} /$ ha.

$\mathrm{ElCO}_{2}$ almacenado en el área de estudio del bosque Tinajillas-Limón Indanza es 17864 t, representando $151 \mathrm{t} / \mathrm{ha}$. Igualmente, en el contenido volumen, carbono almacenado y $\mathrm{CO}_{2}$ la familia y especie con mayor cantidad son Melastomataceae con 46 ton/ha de $\mathrm{CO}_{2}$ almacenado y Miconia sp. con un valor de $31 \mathrm{t} /$ ha de $\mathrm{CO}_{2}$. En la clase I de DAP el $\mathrm{CO}_{2}$ almacenado es $25 \mathrm{t} / \mathrm{ha}$.

\section{Referencias}

Brown, S and FAO. 1997. Estimating Biomass and Biomass Change of Tropical Forests: A Primer. Food and Agriculture Organization of the United Nations. Disponible en: https://goo.gl/kNwLL1.

Corral-Rivas, J, B Vargas-Larreta, C Wehenkel, O Aguirre-Calderón and F. Crecente-Campo. 2013. Guía para el Establecimiento, Seguimiento y Evaluación de Sitios Permanentes de Monitoreo en Paisajes Prodcutivos Forestales. Fondo Sectorial para la Investigación, el Desarrollo y la Innovación Tecnológica Forestal, México. Disponible en: https: //goo.gl/ejiQ3f.

FAO. 2002. "Captura de Carbono en los Suelos para un Mejor Manejo de la Tierra." Organiza- ción de las Naciones Unidas para la Agricultura y la Alimentación. Disponible en: https://goo.gl/ $64 \mathrm{uVDj}$.

FAO. 2004. "Manual de campo: Inventario Forestal Nacional. Guatemala: Programa de Evaluación de los Recursos Forestales." Organización de las Naciones Unidas para la Agricultura y la Alimentación. Disponible en: https://goo.gl/MN7D5J.

FAO. 2006. "Los bosques y el cambio climático." Organización de las Naciones Unidas para la Agricultura y la Alimentación. Disponible en: https: //goo.gl/mgrAX9.

FAO. 2015a. "Manual de Campo: Procedimientos para la planificación, medición y registro de información del Inventario Forestal Nacional del Paraguay." Organización de las Naciones Unidas para la Agricultura y la Alimentación. Disponible en: https://goo.gl/H622xd.

FAO. 2015b. “Términos y definiciones. Actualización de la evaluación de los Recursos Forestales Mundial a 2015." Organización de las Naciones Unidas para la Agricultura y la Alimentación. Disponible en: https:/ /goo.gl/y29SPo.

IPCC. 1995. "Segunda Evaluación. Cambio Climático." Grupo Intergubernamental de expertos sobre el Cambio Climático. Disponible en: https: //goo.gl/K9jKQx.

IPCC. 2006. "Directrices del IPCC para los inventarios nacionales de gases de efecto invernadero. Capítulo 4: Tierras forestales (Volumen 4: Agricultura, silvicultura y otros servicios de la tierra)." Task Force on National Greenhouse Gas Inventories. Disponible en: https://goo.gl/ N4Fdag.

MAE. 2012. Manual de Campo: Evaluación Nacional Forestal. Quito, Ecuador: Ministerio del Ambiente, Ecuador. Disponible en: https://goo.gl/yuzt7p.

Mora, J, N Ulloa, B Quezada and L. López. 2017. "El Carbono Acumulado en la Masa Arbórea de la Reserva Biológica Uyuca, Honduras." Ceiba 54(2):139-146. [en línea] doi: http://dx.doi.org/ 10.5377/ceiba.v54i2.3369.

Pardos, J. A. 2010. Los ecosistemas forestales y el secuestro de carbono ante el calentamiento global. Instituto Nacional de Investigación y Tecnología Agra- 
ria y Alimentaria, Ministerio de Ciencia e Innovación. Madrid - España. Disponible en: https: //goo.gl/FD9PUu.

Riebeek, H. 2005. "The Rising Cost of Natural Hazards." NASA Earth Observatory. Disponible en: https://goo.gl/wiQ81C.

Robertson, N and S. Wunder. 2005. Huellas Frescas en el Bosque. Evaluación de Iniciativas Incipientes de Pagos por Servicios Ambientales en Bolivia. Center for International Forestry Research (CIFOR), Bogor, Indonesia. Disponible en: https://goo.gl/ 3uhkht.

Rügnitz Tito, M, M Chacón León and R. Porro. 2009. Guía para la determinación de carbono en pequeñas propiedades rurales, 1 edición. Lima, Perú: Centro Mundial Agroflorestal (ICRAF) / Consórcio Iniciativa Amazónica. Disponible en: https://goo. gl/7ZX1Q7.

Yáñez Sandoval, A. 2004. “La captura de carbono en bosques: ¿Una Herramienta para la Gestión Ambiental?" Gaceta Ecológica (70):5-18. Disponible en: https:/ /goo.gl/CNDUaN.

Zarco-Espinoza, V. M, J. I Valdez-Hernández, G Ángeles-Pérez and O. Castillo-Acosta. 2010. “Estructura y diversidad de la vegetación arbórea del parque estatal "Agua Blanca", Macuspana, Tabasco." Universidad y Ciencia, Trópico Húmedo 26(1):1-17. Disponible en: https://goo.gl/ QgWndL. 